University of Nebraska - Lincoln

DigitalCommons@University of Nebraska - Lincoln

Faculty Publications from the Department of Engineering Mechanics

Mechanical \& Materials Engineering,

Department of

7-4-2006

\title{
Droplet on a fiber: Geometrical shape and contact angle
}

\author{
Xiangfa Wu \\ Department of Engineering Mechanics, University of Nebraska-Lincoln, xfwu@unlserve.unl.edu
}

Yuris A. Dzenis

Department of Engineering Mechanics, University of Nebraska-Lincoln, ydzenis@unl.edu

Follow this and additional works at: https://digitalcommons.unl.edu/engineeringmechanicsfacpub

Part of the Mechanical Engineering Commons

Wu, Xiangfa and Dzenis, Yuris A., "Droplet on a fiber: Geometrical shape and contact angle" (2006). Faculty Publications from the Department of Engineering Mechanics. 20.

https://digitalcommons.unl.edu/engineeringmechanicsfacpub/20

This Article is brought to you for free and open access by the Mechanical \& Materials Engineering, Department of at DigitalCommons@University of Nebraska - Lincoln. It has been accepted for inclusion in Faculty Publications from the Department of Engineering Mechanics by an authorized administrator of DigitalCommons@University of Nebraska - Lincoln. 
Published in Acta Mechanica 185 (2006), pp. 215-225; doi 10.1007/s00707-006-0349-0

Copyright (C) 2006 Springer-Verlag. Used by permission.

Submitted August 24, 2005; revised March 9, 2006; published online July 4, 2006.

\title{
Droplet on a fiber: Geometrical shape and contact angle
}

\author{
Xiang-Fa Wu and Yuris A. Dzenis \\ Department of Engineering Mechanics, Nebraska Center for Materials and Nanoscience, \\ University of Nebraska-Lincoln, Lincoln, NE 68588-0526, USA \\ Corresponding author - X.-F. Wu, email xfwu@unlserve.unl.edu
}

\begin{abstract}
This paper is concerned with the geometrical shape, wetting length, and contact angle of a microdroplet on a fiber by using the method free energy variation. The governing equation and relevant boundary conditions of the microdroplet were re-derived based on the free energy variation of the droplet/fiber system. The geometrical shape of the droplet was determined as the combination of Legendre's elliptical functions of the first and second kinds, corresponding to the previous results in literature [6]. For contact angle $\theta$ $>15^{\circ}$, a novel efficient semi-analytic approach was proposed to extract the contact angle from experimental data. The given approach can be used as theoretical basis of determining surface tension of fluids based on a sessile drop on a fiber.
\end{abstract}

\section{Introduction}

Wetting properties of fluids on fiber surfaces play an important role in the textile industry and polymer composites engineering. In textile industry people mainly consider the nature of fiber dyeing and cleaning properties by surfactants and the ability of substances to waterproof fabrics. In polymer composites engineering, researchers have paid significant attention to the interface properties of various fiber/matrix systems such as the fiber surface wetting property and the fiber/matrix interface bonding strength among others. These properties affect the toughening and damage mechanisms of fibers as reinforcement in composite materials [1]. As one of the typical micromechanical testing methods, the fiber microdebond test has been developed for evaluating the fiber/matrix interface strength [2], [3]. In this testing process, fabrication of uniform micro samples with polymer microdroplets on monofilaments is the key step to yield repeatable experimental data since the geometrical shape, aspect ratio, and contact angle of the droplet affect the test results. As a matter of fact, the geometry of a droplet on a fiber is governed by the wetting property of the droplet.

The wetting property of microdroplets on monofilaments has attracted remarkable attention in decades [4]. The wetting shape of a microdroplet on a fiber does not appear as a partial sphere as that on a flat surface. Within the framework of Laplace's formula of surface tension and Young's equation of wetting angle, Yamaki and Katayama [5] first studied the shape of a microdroplet on a fiber by solving the governing equation numerically. Carroll [6] considered this problem through an analytic approach, and he found that the droplet shape could be expressed as the combination of Legendre's elliptical functions of the first 
and second kinds. Furthermore, in an attempt to simplify the use of Carroll's formula in practice, Wagner [7] and Song et al. [8] introduced quite a few numerical algorithms to invert Carroll's integrations and then to extract the contact angle numerically from experimental data. Their results highly depend upon the accuracy of the numerical calculation of the incomplete Legendre's elliptic integrals, Carson's integrals of the first and the second kinds, and the numerical solutions of algebraic equations involving trigonometrical functions. These processes are still relatively complicated for users in practice.

Therefore, in this work we further investigate the above problem theoretically. Different from the previous works [5]-[8], we begin with the free energy variation of a microdroplet on a homogenous cylindrical fiber. Without the aid of any further assumptions, we derive the exact governing equation and correct boundary conditions governing the geometrical shape, fiber wetting length, and contact angle of the microdroplet. In an attempt to extract the contact angle from experimental data, a novel efficient semi-analytic approach with very high accuracy is proposed in the middle range of contact angle $\left(\theta>15^{\circ}\right)$. The potential applications of the model were further addressed.

\section{Problem formulation and solution procedure}

Generally, a liquid placed on a flat solid surface will form a drop with a definite contact angle between the liquid and solid phase as shown in Figure 1. The drop/surface wetting properties can be determined [9][11] such that:

Nonwetting in the case of $\gamma_{\mathrm{LS}}>\gamma_{\mathrm{SV}}+\gamma \cos \theta$, for any value of $\theta \in\left[0,180^{\circ}\right]$,

Partial wetting in the case of $\gamma_{S V}=\gamma_{L S}+\gamma \cos \theta$, for a specific value of $\theta \in\left[0,180^{\circ}\right]$,

Complete wetting in the case of $\gamma_{\mathrm{SV}}>\gamma_{\mathrm{LS}}+\gamma \cos \theta$, for any value of $\theta \in\left[0,180^{\circ}\right]$,

where $\gamma_{\mathrm{SV}}$ is the solid-vapor surface tension (specific surface energy), $\gamma$ is the liquid-vapor surface tension (surface tension), and $\gamma_{L S}$ is the liquid-solid surface tension (interfacial tension). In case (1) the liquid should not wet the solid at all; in case (2) the three surface tensions balance along the line of mutual contact to form the contact angle $\theta$; and in case (3) the Gibbs function is minimized when the solid is wetted completely. In the equilibrium condition with other forces ignored (such as force of gravity, chemical, electromagnetic, and hydrostatic forces, etc.), according to Young's equation (2), the contact angle $\theta$ can be used as a measure of the interfacial tension when the two other interfacial tensions are known. For a microdroplet, the effect of its gravity on the droplet shape is negligible. The microdroplet forms a partial sphere when placed on a flat surface [10], while it forms a bell-shaped surface when placed on a cylindrical fiber. Figure 2 shows a typical epoxy microdroplet placed on a $\mathrm{SiC}$ fiber after curing [12], which was used as the microdebond specimen for evaluating the interface strength between epoxy and fiber.

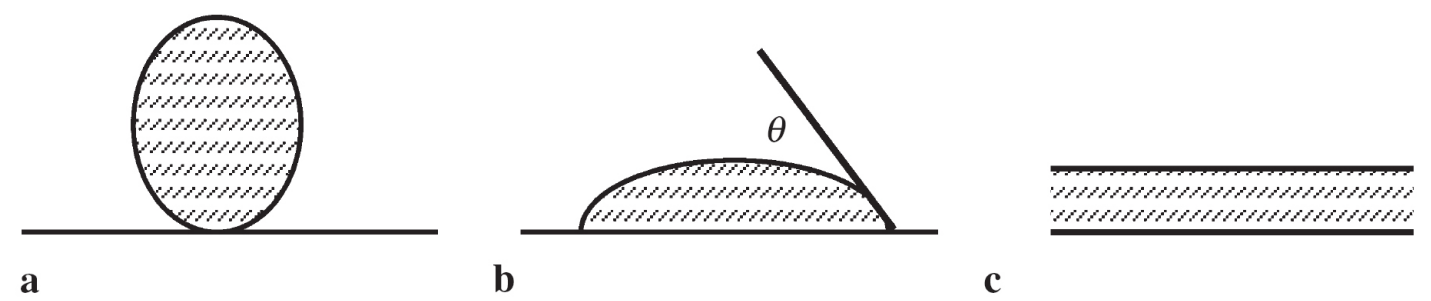

Figure 1. Different configurations of the vapor-fluid-solid interfaces: a) nonwetting; b) partial wetting; c) complete wetting. 

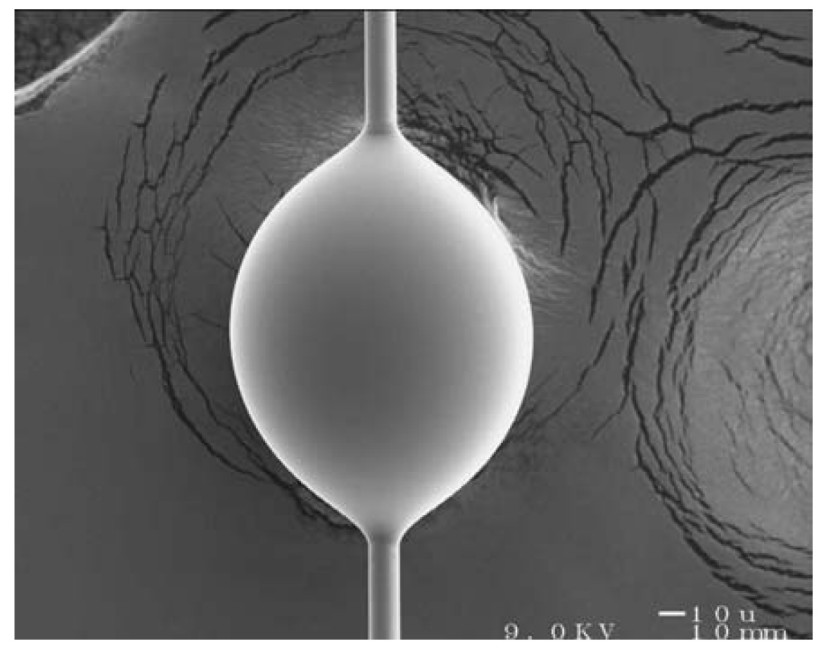

Figure 2. SEM micrograph of an epoxy microdroplet on a $\mathrm{SiC}$ fiber (fiber diameter $\sim 14^{\circ} \mu \mathrm{m}$ ) [12].

Now let us consider a microdroplet placed on a homogenous cylindrical fiber. When the microdroplet is in equilibrium, its surface forms a surface of revolution with the axisymmetric $x$-axis as shown in Figure 3 . According to the wetting theories [13]-[14], the total free energy of the microdroplet can be expressed by

$$
J=4 \pi \int_{0}^{L / 2}\left\{\left(\gamma_{\mathrm{LS}}-\gamma_{\mathrm{SV}}\right) \gamma_{0}+\gamma y(x) \sqrt{1+\left[y^{\prime}(x)\right]^{2}}\right\} d x
$$

where the geometrical symmetry of the microdroplet has been considered, and the force of gravity and other forces such as chemical, electromagnetic, and hydrostatic forces are ignored. In relation (4) $y(x)$ is

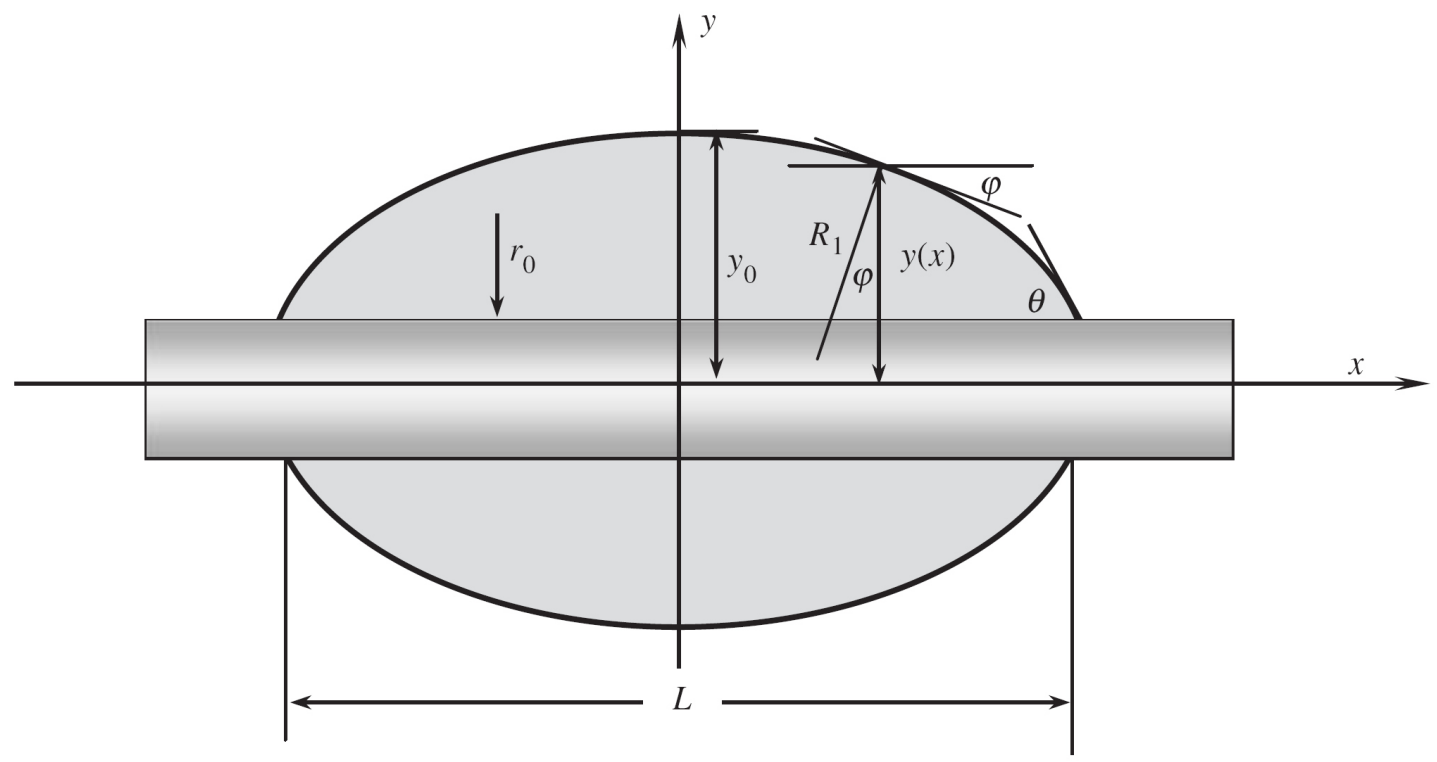

Figure 3. Schematic diagram of a microdroplet on a fiber. 
the microdroplet radius at locus $x$. The known boundary conditions at $x=0$ and $x=L / 2$ are

$$
y^{\prime}(0)=0, \quad y(L / 2)=r_{0}
$$

The unknown conditions are the microdroplet peak radius $y(0)$ and the fiber wetting length $L$, which will be determined according to the volume conservation of the microdroplet:

$$
V_{0}=2 \pi \int_{0}^{L / 2}\left\{[y(x)]^{2}-r_{0}^{2}\right\} d x,
$$

where $V_{0}$ is the microdroplet volume, a constant for a given microdroplet/fiber system, and $r_{0}$ is the fiber radius.

For a given microdroplet in equilibrium on a fiber, the free energy functional (4) should take the extremum under the conditions (5) and (6). This variational problem under the subsidiary conditions can be reformulated into an unconditioned variational problem by introducing Lagrange's multiplier $\mu$ :

$$
\begin{aligned}
\Pi & =J-\mu V_{0} \\
& =4 \pi \int_{0}^{L / 2} F\left[y(x), y^{\prime}(x), \mu\right] d x,
\end{aligned}
$$

where:

$$
F\left[y(x), y^{\prime}(x), \mu\right]=\left(\gamma_{L S}-\gamma_{S V}\right) r_{0}+\gamma y(x) \sqrt{1+\left[y^{\prime}(x)\right]^{2}}-\mu\left\{[y(x)]^{2}-r_{0}^{2}\right\} / 2,
$$

with two ends on the $x$ - and $y$-axes satisfying Equations (5), respectively. The extremal of the free energy functional (7) leads to the classic Euler-Lagrange equation

$$
F_{y}-d F_{y^{\prime}} d x=0,
$$

with two transversality conditions at $x$ - and $y$-axes:

$$
F_{y^{\prime} \mid x=0}=0
$$

and

$$
F-\left.F_{y^{\prime}} y^{\prime}\right|_{x=L / 2}=0 \text {. }
$$

Let us first consider these transversality conditions. Substitution of Equation (8) into (10) yields

$$
y(0) y^{\prime}(0) / \sqrt{1+\left[y^{\prime}(0)\right]^{2}}=0,
$$

which accords to the symmetric condition (5) at $x=0$. Furthermore, plugging Equation (8) into (11) leads to

$$
\left(\gamma_{L S}-\gamma_{S V}\right) r_{0}-\mu\left\{[y(L / 2)]^{2}-r_{0}^{2}\right\} / 2+\gamma y(L / 2) / \sqrt{1+\left[y^{\prime}(2)\right]^{2}}=0 .
$$

With the aid of relation (5), i.e., $y(L / 2)=r_{0^{\prime}}$ and the definition of the contact angle $h$ at $x=L / 2$, 


$$
y^{\prime}(L / 2)=-\tan \theta
$$

Equation (13) leads to the contact angle of the microdroplet on the fiber:

$$
\cos \theta=\left(\gamma_{\mathrm{SV}}-\gamma_{\mathrm{LS}}\right) / \gamma
$$

which is constant for a given microdroplet/fiber system. Equation (15) is exactly Young's equation of contact angle. This shows that the contact angle of a microdroplet on a fiber is a material constant, independent of the geometrical aspect ratio and equal to that on a flat surface. This confirms the choice of constant contact angle in previous works [5], [6].

Now let us further consider the Euler-Lagrange equation (9). Substitution of Equation (8) into (9) yields

$$
\frac{d}{d x} \frac{y(x) y^{\prime}(x)}{\sqrt{1+\left[y^{\prime}(x)\right]^{2}}}-\sqrt{1+\left[y^{\prime}(x)\right]^{2}}+\frac{\mu}{\gamma} y(x)=0,
$$

which may be reformulated as

$$
\gamma\left\{1 /\left\{y(x) \sqrt{1+\left[y^{\prime}(x)\right]^{2}}\right\}-y^{\prime \prime}(x) / \sqrt[3]{\left\{1+\left[y^{\prime}(x)\right]^{2}\right\}^{2}}\right\}=\mu .
$$

Due to the revolution surface of the microdroplet shown in Figure 3, the radii of curvature of the surface at locus $[x, y(x)]$ can be expressed by

$$
\begin{aligned}
& 1 / R_{1}=1 /(y \operatorname{cosec} \varphi)=1 /\left[y(x) \sqrt{1+\left[y^{\prime}(x)\right]^{2}}\right] \\
& 1 / R_{2}=-y^{\prime \prime}(x) / \sqrt[3]{\left\{1+\left[y^{\prime}(x)\right]^{2}\right\}^{2}} .
\end{aligned}
$$

Therefore, relation (17) can be recast as

$$
\gamma\left(1 / R_{1}+1 / R_{2}\right)=\mu
$$

where $\mu$ is Lagrange's multiplier, a constant. Equation (19) is the familiar Laplace's formula of surface tension. Lagrange's multiplier $\mu$ with pressure unit can be expressed such that

$$
\mu=\Delta p=p_{L}-p_{V}
$$

where $p_{L}$ and $p_{V}$ are the pressures inside and outside the microdroplet, respectively.

The microdroplet shape shown in Figure 3 can be determined by solving the differential equation (16) [6]. With conditions at $x=0$ and $x=L / 2$, it can be easily proved that Equation (16) has the first integration

$$
1 / \sqrt{1+\left[y^{\prime}(x)\right]^{2}}=A y(x)+B / y(x),
$$

where

$$
A=\mu / 2 \gamma \theta=\left(y_{0}-r_{0} \cos \theta\right) / y_{0}{ }^{2}-r_{0}{ }^{2}, B=y_{0} r_{0}\left(y_{0} \cos \theta-\mathrm{r}_{0}\right)=\left(y_{0}{ }^{2}-r_{0}{ }^{2}\right) \text {. }
$$

The excess pressure of the microdroplet can be determined from Equations (20) and (22) as

$$
\Delta p=p_{L}-p_{V}=2 \gamma\left(y_{0}-r_{0} \cos \theta\right) /\left(y_{0}^{2}-r_{0}^{2}\right) \text {. }
$$


Furthermore, the microdroplet shape can be obtained by integrating Equation (21):

$$
x=\int_{y}^{y_{0}}(A y+B / y) / \sqrt{1-[A y+B / y]^{2}} d y .
$$

Substitution of Equation (22) into (24) leads to a concise form:

$$
x=\int_{y}^{y_{0}}\left(y^{2}+\lambda r_{0} y_{0}\right) / \sqrt{\left(y_{0}^{2}-y^{2}\right)\left(y^{2}-\lambda^{2} r_{0}^{2}\right)} d y,
$$

with

$$
\lambda=\left(y_{0} \cos \theta-r_{0}\right) /\left(y_{0}-r_{0} \cos \theta\right) .
$$

By using the variable transformation

$$
y^{2}=y_{0}^{2}\left(1-k^{2} \sin ^{2} \varphi\right)
$$

with

$$
k^{2}=1-\lambda^{2}\left(r_{0} / y_{0}\right)^{2}
$$

the integral (25) can be expressed in terms of two special functions [6]:

$$
x=\lambda r_{0} F(k, \varphi)+y_{0} E(k, \varphi),
$$

where $F(k, \varphi)$ and $E(k, \varphi)$ are respectively Legendre's elliptical functions of the first and second kinds defined as

$$
F(k, \varphi)=\int_{0}^{\varphi}\left(1-k^{2} \sin ^{2} \phi\right)^{-1 / 2} d \phi, \quad E(k, \varphi)=\int_{0}^{\varphi} \sqrt{1-k^{2} \sin ^{2} \phi} d \phi \quad(k \leq 1) .
$$

The fiber wetting length $L$ can be obtained by using condition (25) of $y(L / 2)=r_{0}$ such that

$$
L=2\left[\lambda r_{0} F\left(k, \varphi_{0}\right)+y_{0} E\left(k, \varphi_{0}\right)\right]
$$

where $\varphi_{0}$ is the dimensionless parameter determined from Equation (27) with $y=r_{0}$. Consequently, the volume of the microdroplet can be expressed as

$$
\begin{aligned}
V_{0}= & 2 \pi \int_{r_{0}}^{y_{0}} y^{2}\left(y^{2}+\lambda r_{0} y_{0}\right) / \sqrt{\left(y_{0}^{2}-y^{2}\right)\left(y^{2}-\lambda^{2} r_{0}^{2}\right)} d y-\pi r_{0}^{2} L \\
= & \frac{2 \pi y_{0}}{3}\left[\left(2 \lambda^{2} r_{0}^{2}+3 \lambda r_{0} y_{0}+2 y_{0}^{2}\right) E\left(k, \varphi_{0}\right)-\lambda^{2} r_{0}^{2} F\left(k, \varphi_{0}\right)\right. \\
& \left.+r_{0}^{2}\left(r_{0} / y_{0}\right) \sqrt{\left(y_{0}^{2}-x_{0}^{2}\right)\left(1-\lambda^{2}\right)}\right]-\pi r_{0}^{2} L .
\end{aligned}
$$

Therefore, for a given microdroplet of volume $V_{0}$ on a fiber of radius $r_{0}$, the droplet geometries $\theta$, $L$, and $y_{0}$ are determined completely by the system. Once $L$ and $y_{0}$ are measured in experiments, the contact angle $\theta$ can be calculated by the inverse of Equation (31), which can be solved numerically referring to the work by Carroll [6], Wagner [7], and Song et al. [8], among others. Once the contact angle $\theta$ is determined, Equations (29), (31), and (32) determine the microdroplet shape, wetting length, and volume completely for any given aspect ratios. 


\section{Contact angle of microdroplet on a fiber}

In practice, the microdroplet geometries $L, y_{0}$, and $r_{0}$ are usually measured by means of optical microscopy and electron scanning microscopy (SEM). Once $L, y_{0}$, and $r_{0}$ are available, the contact angle $\theta$ can be approximated through matching Carroll's characteristic curves [6]. However, it has been shown that an accuracy better than $\pm 5^{\circ}$ cannot be obtained based on this method. In an attempt to overcome the limitation of Carroll's work, Wagner [7], Song et al. [8], and others numerically inverted Equation (31) to extract the characteristic $\theta-\left(L / r_{0}\right)$ curves for various aspect ratios $\left(y_{0} / r_{0}\right)$ that appear more convenient for practical use. Here we propose an efficient semi-analytic approach to simplify the inverse of Equation (31) in the middle range of contact angle $\left(\theta>15^{\circ}\right)$. As a matter of fact, the dimensionless wetting length $l$ (Equation (25) at $x=L / 2$ ) may be expressed into a function of a dimensionless variable $\lambda$ :

$$
l(\lambda)=\int_{1}^{\rho_{0}}\left(\rho^{2}+\lambda \rho_{0}\right) / \sqrt{\left(\rho_{0}^{2}-\rho^{2}\right)\left(\rho^{2}-\lambda^{2}\right)} d \rho,
$$

where the dimensionless variables are

$$
l(\lambda)=L /\left(2 r_{0}\right), \quad \rho=y / r_{0}, \quad \rho_{0}=y_{0} / r_{0}, \quad \lambda=\frac{y_{0} \cos \theta-r_{0}}{y_{0}-\cos \theta}=\frac{\rho_{0} \cos \theta-1}{\rho_{0}-\cos \theta} .
$$

Here the magnitude of $\lambda$ is no more than 1.0 for a realistic microdroplet/fiber system, and the aspect ratio $\rho_{0}$ is always larger than 1.0. Introduce the following Taylor's series expansion based on Newton's binominal formula:

$$
\frac{1}{\sqrt{1-(\lambda / \rho)^{2}}}=1+\frac{1}{2}\left(\frac{\lambda}{\rho}\right)^{2}+\frac{3}{8}\left(\frac{\lambda}{\rho}\right)^{4}+\frac{5}{16}\left(\frac{\lambda}{\rho}\right)^{6}+\frac{35}{64}\left(\frac{\lambda}{\rho}\right)^{8}+\frac{231}{512}\left(\frac{\lambda}{\rho}\right)^{10} \ldots
$$

which decays rapidly for $(\lambda / \rho)<1.0$. Substitution of Equation (35) into (33) leads to an approximate expression of $l(\lambda)$ :

$$
\begin{aligned}
l(\lambda) \approx & \int_{1}^{\rho_{0}} \frac{1}{\rho \sqrt{\left(\rho_{0}^{2}-\rho^{2}\right)}}\left[\rho^{2}+\rho_{0} \lambda+\frac{1}{2} \lambda^{2}+\frac{\rho_{0}}{2} \frac{\lambda^{3}}{\rho^{2}}+\frac{3}{8} \frac{\lambda^{4}}{\rho^{2}}\right. \\
& \left.+\frac{3 \rho_{0}}{8} \frac{\lambda^{5}}{\rho^{4}}+\frac{5}{16} \frac{\lambda^{6}}{\rho^{4}}+\frac{5 \rho_{0}}{16} \frac{\lambda^{7}}{\rho^{6}}+\frac{35}{128} \frac{\lambda^{8}}{\rho^{6}}+\frac{35 \rho_{0}}{128} \frac{\lambda^{9}}{\rho^{10}}+\frac{63}{256} \frac{\lambda^{10}}{\rho^{10}}+\frac{63 \rho_{0}}{256} \frac{\lambda^{11}}{\rho^{12}}+\frac{231}{512} \frac{\lambda^{12}}{\rho^{12}}\right] d \rho .
\end{aligned}
$$

The integration of each term in Equation (36) can be expressed explicitly in terms of fundamental functions. As a result, a polynomial approach of $l(\lambda)$ can be obtained:

$$
\begin{aligned}
l(\lambda)=C_{0}+C_{1} \lambda & +C_{2} \lambda^{2}+C_{3} \lambda^{3}+C_{4} \lambda^{4}+C_{5} \lambda^{5}+C_{6} \lambda^{6}+C_{7} \lambda^{7}+C_{8} \lambda^{8} \\
& +C_{9} \lambda^{9}+C_{10} \lambda^{10}+C_{11} \lambda^{11}++C_{12} \lambda^{12}
\end{aligned}
$$

where 


$$
\begin{aligned}
& C_{0}=\sqrt{\rho_{0}^{2}-1}, \quad C_{1}=-\ln \left(\rho_{0}-\sqrt{\rho_{0}^{2}-1}\right), \quad C_{2}=-\frac{1}{2 \rho_{0}} \ln \left(\rho_{0}-\sqrt{\rho_{0}^{2}-1}\right), \\
& C_{3}=\frac{1}{2 \rho_{0}^{2}}\left[\frac{\rho_{0}}{2} \sqrt{\rho_{0}^{2}-1}-\frac{1}{2} \ln \left(\rho_{0}-\sqrt{\rho_{0}^{2}-1}\right)\right], \quad C_{4}=\frac{3}{8 \rho_{0}^{3}}\left[\frac{\rho_{0}}{2} \sqrt{\rho_{0}^{2}-1}-\frac{1}{2} \ln \left(\rho_{0}-\sqrt{\rho_{0}^{2}-1}\right)\right], \\
& C_{5}=\frac{3}{8 \rho_{0}^{4}}\left[\frac{\rho_{0}^{3}}{4} \sqrt{\rho_{0}^{2}-1}+\frac{3 \rho_{0}}{8} \sqrt{\rho_{0}^{2}-1}-\frac{3}{8} \ln \left(\rho_{0}-\sqrt{\rho_{0}^{2}-1}\right)\right] \\
& C_{6}=\frac{5}{16 \rho_{0}^{5}}\left[\frac{\rho_{0}^{3}}{4} \sqrt{\rho_{0}^{2}-1}+\frac{3 \rho_{0}}{8} \sqrt{\rho_{0}^{2}-1}-\frac{3}{8} \ln \left(\rho_{0}-\sqrt{\rho_{0}^{2}-1}\right)\right], \\
& C_{7}=\frac{5}{16 \rho_{0}^{6}}\left[\frac{\rho_{0}^{5}}{6} \sqrt{\rho_{0}^{2}-1}+\frac{5 \rho_{0}^{3} \sqrt{\rho_{0}^{2}-1}}{24}+\frac{5 \rho_{0}}{16} \sqrt{\rho_{0}^{2}-1}-\frac{5}{16} \ln \left(\rho_{0}-\sqrt{\rho_{0}^{2}-1}\right)\right], \\
& C_{8}=\frac{35}{128 \rho_{0}^{7}}\left[\frac{\rho_{0}^{5}}{6} \sqrt{\rho_{0}^{2}-1}+\frac{5 \rho_{0}^{3} \sqrt{\rho_{0}^{2}-1}}{24}+\frac{5 \rho_{0}}{16} \sqrt{\rho_{0}^{2}-1}-\frac{5}{16} \ln \left(\rho_{0}-\sqrt{\rho_{0}^{2}-1}\right)\right], \\
& C_{9}=\frac{63}{128 \rho_{0}^{8}}\left[\frac{\rho_{0}^{7}}{8} \sqrt{\rho_{0}^{2}-1}+\frac{7 \rho_{0}^{5}}{48} \sqrt{\rho_{0}^{2}-1}+\frac{35 \rho_{0}^{3} \sqrt{\rho_{0}^{2}-1}}{192}+\frac{35 \rho_{0}}{128} \sqrt{\rho_{0}^{2}-1}-\frac{35}{128} \ln \left(\rho_{0}-\sqrt{\rho_{0}^{2}-1}\right)\right], \\
& C_{10}=\frac{63}{256 \rho_{0}^{9}}\left[\frac{\rho_{0}^{7}}{8} \sqrt{\rho_{0}^{2}-1}+\frac{7 \rho_{0}^{5}}{48} \sqrt{\rho_{0}^{2}-1}+\frac{35 \rho_{0}^{3} \sqrt{\rho_{0}^{2}-1}}{208}+\frac{35 \rho_{0}}{128} \sqrt{\rho_{0}^{2}-1}-\frac{35}{128} \ln \left(\rho_{0}-\sqrt{\rho_{0}^{2}-1}\right)\right], \\
& C_{11}=\frac{231}{512 \rho_{0}^{10}}\left[\frac{\rho_{0}^{9}}{10} \sqrt{\rho_{0}^{2}-1}+\frac{9 \rho_{0}^{7}}{80} \sqrt{\rho_{0}^{2}-1}+\frac{21 \rho_{0}^{5}}{160} \sqrt{\rho_{0}^{2}-1}+\frac{63 \rho_{0}^{3} \sqrt{\rho_{0}^{2}-1}}{504}\right. \\
& \left.+\frac{63 \rho_{0}}{256} \sqrt{\rho_{0}^{2}-1}-\frac{63}{256} \ln \left(\rho_{0}-\sqrt{\rho_{0}^{2}-1}\right)\right] \\
& C_{12}=\frac{231}{512 \rho_{0}^{11}}\left[\frac{\rho_{0}^{9}}{10} \sqrt{\rho_{0}^{2}-1}+\frac{9 \rho_{0}^{7}}{80} \sqrt{\rho_{0}^{2}-1}+\frac{21 \rho_{0}^{5}}{160} \sqrt{\rho_{0}^{2}-1}+\frac{63 \rho_{0}^{3} \sqrt{\rho_{0}^{2}-1}}{504}\right. \\
& \left.+\frac{63 \rho_{0}}{256} \sqrt{\rho_{0}^{2}-1}-\frac{63}{256} \ln \left(\rho_{0}-\sqrt{\rho_{0}^{2}-1}\right)\right] .
\end{aligned}
$$

It can be observed that $l(\lambda)$ in Equation (33) increases monotonically in the domain $\lambda \in(0,1)$ since its first derivative

$$
l^{\prime}(\lambda)=\int_{1}^{\rho_{0}} \rho_{0} / \sqrt{\left(\rho_{0}^{2}-\rho^{2}\right)\left(\rho^{2}-\lambda^{2}\right)} d \rho+\lambda \int_{1}^{\rho_{0}}\left(\rho^{2}+\lambda \rho_{0}\right) / \sqrt{\left(\rho_{0}^{2}-\rho^{2}\right)\left(\rho^{2}-\lambda^{2}\right)^{3}} d \rho
$$

is always positive in this domain, and also the coefficients of polynomial $(37) C_{i}(i=1,2, \ldots, 12)$ are all positive. Therefore, Equation (37) only has one single root $\lambda_{0}$ in this domain for a given $l_{0}$, which can be easily 


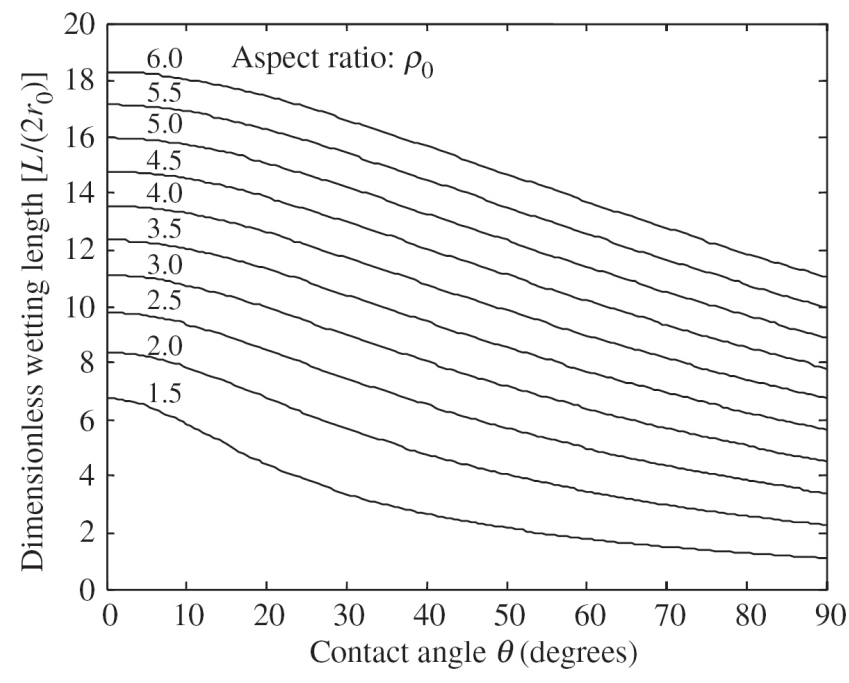

Figure 4. Variation of contact angle with dimensionless length $\left[L /\left(2 r_{0}\right)\right]$ for different aspect ratios $\left(\rho_{0}\right)$.

determined using existing numerical algorithms such as the simple Newton's method. Finally, the contact angle $\theta$ can be extracted from Equation (34):

$$
\theta=\cos ^{-1}\left[\left(1+\lambda_{0} \rho_{0}\right) /\left(\lambda_{0}+\rho_{0}\right)\right] .
$$

With the aid of relations (34), (37), (38), and (40), a family of characteristic $l-\theta$ curves for several aspect ratios $\rho_{0}$ are plotted in Figure 4 , which provide a very precise approach for contact angle $\theta>15^{\circ}$.

As an example to illustrate the accuracy of the present method, we choose the experimental data for the contact angle of glycerol droplets on carbon filaments as shown in Table 1 [6]. It can be found that, in the middle range of contact angles as discussed, results predicted by the present method (Column D in Table 1) are extremely close to those obtained by Wagner's methods (Columns B and C in Table 1). When $\theta$ is close to $0^{\circ}$ and therefore $\lambda$ very close to 1 , the present method may lead to cutoff errors due to use of the cutoff Taylor's series (35). Nevertheless, numerical experiments show that at $\theta>15^{\circ}$ the cutoff error is lower than $0.5^{\circ}$. Therefore, the present method is sufficient for most of practical use usually with contact angle $\theta>15^{\circ}$.

Table 1. Contact angle of glycerol droplets on carbon filaments

\begin{tabular}{llccccc}
\hline $\begin{array}{l}\text { Droplet } \\
\text { number }\end{array}$ & $\begin{array}{l}\text { Dimensionless } \\
\text { droplet length }\end{array}$ & $\begin{array}{c}\text { Aspect } \\
\text { ratio }\end{array}$ & \multicolumn{2}{l}{ Contact angle $(\theta)$ (degrees) } \\
\cline { 5 - 7 } \cline { 4 - 6 } & $(l)$ & & $A$ & $B$ & $C$ & $D$ \\
\hline 1 & 10.00 & 4.00 & $\sim 45-50$ & 48.46 & 48.42 & 48.425 \\
2 & 10.00 & 4.75 & $\sim 67-72$ & 69.20 & 69.20 & 69.209 \\
3 & 10.00 & 4.13 & $\sim 48-53$ & 51.87 & 51.93 & 52.072 \\
4 & 12.57 & 5.00 & $\sim 44-49$ & 47.36 & 47.45 & 47.470 \\
5 & 11.43 & 4.57 & $\sim 45-50$ & 48.38 & 48.49 & 48.438 \\
6 & 10.86 & 4.43 & $\sim 47-53$ & 50.83 & 50.85 & 50.853 \\
7 & 12.57 & 5.14 & $\sim 48-54$ & 51.01 & 51.06 & 51.007 \\
8 & 9.43 & 3.57 & $\sim 40-45$ & 42.43 & 42.39 & 42.329 \\
9 & 9.00 & 3.57 & $\sim 45-50$ & 47.07 & 47.09 & 47.056 \\
\hline
\end{tabular}

Experimental data by Wagner (1990).

$A$ : Data predicted by Carroll's method [6].

$B$ : Data predicted by Wagner's method [7].

$C$ : Data predicted by Wagner's method [7].

$D$ : Data predicted using the present method. 


\section{Concluding remarks}

The governing equation and exact boundary conditions for determining the geometrical shape and contact angle of a microdroplet on a fiber have been obtained by means of the variation of the free energy functional. The geometrical shape, fiber wetting length, and microdroplet volume are determined in closedform. In an attempt to extract the contact angle precisely in practice, an novel efficient numerical approach is proposed by expressing the fiber wetting length in terms of a series expansion of the characteristic number $\lambda$, which gives very high accuracy for contact angle $\theta>15^{\circ}$.

The present method is based on the principle of energy variation. Therefore, it is convenient to further include effects of other forces on the geometrical shape of microdroplets placed on complex surfaces. In these cases, the free energy functional can be used directly for developing the relevant finite element method (FEM) and boundary element method (BEM) for microdroplet systems. The approach given in this work can be used for the scaling analysis of the microdroplet aspect ratio and contact angle. The present method can be used with great advantage in understanding of the wettability of fluid droplets on filaments, which are used extensively in the textile industry and fiber/epoxy composites engineering.

\section{Acknowledgments}

Partial support of this work by the U. S. Army Research Office and the U. S. Air Force Office of Scientific Research is gratefully acknowledged. The authors would like to thank the anonymous reviewers for Acta Mechanica for helpful comments and suggestions to improve the present version.

\section{References}

[1] Kim, J. K., Mai, Y. M.: Engineered interfaces in fiber reinforced composites. New York: Elsevier 1998.

[2] Miller, B., Gaur, U., Hirt, D. E.: Measurement and mechanical aspects of the microbond pull-out technique for obtaining fiber resin interfacial shear-strength. Compos. Sci. Tech. 42, 207-219 (1991).

[3] Miller, B., Muri, P. L.: Microbond method for determination of the shear strength of a fiber/resin interface. Compos. Sci. Tech. 28, 17-32 (1987).

[4] Demarquette, N. R.: Evaluation of experimental techniques for determining interfacial tension between molten polymers. Int. Mater. Rev. 48, 247-269 (2003).

[5] Yamaki, J. I., Katayama, Y.: New method of determining contact angle between monofilament and liquid. J. Appl. Polym. Sci. 19, 2897-2909 (1975).

[6] Carroll, B. J.: The accurate measurement of contact angle, phase contact area, drop volume, and Laplace excess pressure in drop-on-fiber system. J. Colloid Interface Sci. 57, 488-495 (1976).

[7] Wagner, H. D.: Spreading of liquid droplets on cylindrical surface-Accurate determination of contact angle. J. Appl. Phys. 67, 1352-1355 (1990).

[8] Song, B. H., Bismarck, A., Tahhan, R., Springer, J.: A generalized drop length-height method for determination of contact angle in drop-on-fiber system. J. Colloid Interface Sci. 197, 68-77 (1998). 
[9] Adamson, A. W., Gast, A. P.: Physical chemistry of surfaces, 6th ed. New York: John Wiley \& Sons 1997.

[10] Hudson, J. B.: Surface science: An introduction. New York: John Wiley \& Sons (1998).

[11] Israelachvili, J.: Intermolecular \& surface forces, 2nd ed. New York: Academic Press 2003.

[12] Petersen, B. T.: Advanced composites with fiber matrix interfaces modified with nanoparticles (MS Thesis). Lincoln: University of Nebraska 2003.

[13] Blokhuis, E. M., Shilkrot, Y., Widom, B.: Young's law with gravity. Molecular Phys. 86, 891-899 (1995).

[14] Safran, S. A.: Statistical thermodynamics of surfaces, interfaces, and membranes. Cambridge, Mass.: Westview Press 2003. 